\title{
Improving Power Flow Control in AC Transmission System Using Phase Shifting Transformer (PST)
}

\author{
Sochima Vincent Egoigwe ${ }^{1}$ Timothy Oluwaseun Araoye ${ }^{1^{*}} \quad$ Frederick Udebunu Ilo $^{2}$ \\ 1.Department of Mechatronics Engineering, University of Nigeria, Nsukka, Nigeria \\ 2.Department of Electrical and Electronic Engineering, Enugu State University of Science and Technology, \\ Enugu, Nigeria
}

\begin{abstract}
Obtaining optimal power flow has been a challenge over the years for power system engineers. Several ways and technologies ranging from conventional to the use of modern Flexible Alternating Current Transmission Systems (FACTS) devices have been incorporated into the power system to ensure optimal flow of power. FACTS devices used are capable of mitigating several power system problems ranging from line overloading, voltage instability, transient instability, and Power system congestion and so on. Phase shifting transformer (PST) is a FACTS device that is capable of controlling power flow problems in a network by varying its phase angle. PST, when installed at appropriate locations on the power system is capable of redirecting and redistributing power flow from overloaded lines to the less loaded ones, hence ensuring an optimal performance and improved performance of the power system.This paper evaluates the effect of incorporating the PST on the IEEE 5 bus system. Newton-Raphson load flow technique is employed in modeling the IEEE 5 bus system and the PST. Simulation was carried out using MATLAB software package for both the steady state condition and with the incorporation of PST. Results showed that overloading on some transmission lines was checked and mitigated by redistribution of active power across the system. There was also reduction of losses on the lines.
\end{abstract}

Keywords: Phase Shifting Transformer, Power System, Power flow, MATLAB, Newton-Raphson.

DOI: $10.7176 /$ CEIS/11-4-03

Publication date:June $30^{\text {th }} 2020$

\subsection{INTRODUCTION}

Over the years, power system security has been constantly threatened as a result of an ever increasing load being added to the system, failure of one or more system components, transients. Increased power demand poses serious challenge to the load handling capability of the power system as an outage of transmission line failure may result in overload on other lines [16]. Consequently, this may have a cascaded failure of the transmission lines, hence, resulting in partial or total system collapse.

Overloading on of transmission lines in the power system can be mitigated by appropriate redistribution of power flow from the overloaded lines to the less loaded lines. One of the effective techniques used to control power flow in a system is the installation of phase shifting transformers (PST) on appropriate lines. PST is FACTS device controls the active power flow by means of varying its phase angle between the primary side voltage and the secondary side voltage. This helps to regulate the flow of power on the lines and between the buses in the power system [14]. This phase shift of the PST controls the active power flow, both in magnitude and direction [1].

Power flow analysis is mostly important for system planning and operation in order to improve power quality. They notify and detect components that are over-loaded in the voltage profiles. Also, power flow system can be applied to analysis reliability index and forecast the future load demand in the system. $[6,13]$. The highly common methods of power flow are Gauss-Seidel and Newton-Raphson which are widely used in transmission systems but produce worse robustness and performance when applied to distribution system [5]. This occurs due to distribution nature of the network which is design through feebly meshed topology or a dial and very high $\mathrm{R} / \mathrm{X}$ ratio. There are many proposed methods to minimized these problems, such as backward-forward sweep and Z bus Gauss techniques $[12,19]$. Moreover the direct approach (DA) also proposed which is the very efficient and unique [20]. The direct approach (DA) technique save time for performing LU factorization which involves backward and forward substitution of the admittance matrices or jacobian, which are commonly manual formulations. Thus, the design of direct approach (DA) allows the application of real-time in the smart grid. Therefore, reference [4] used DA methods to analyze optimal power flow (OPF) in FACTS distribution network for industrial grid.

In reference [3] the three- phase method applied perform sequentially the mutual couplings and selfimpedances for the operation but shunt admittances are nullified. The lines system design is always short-length and non-tapped transformers even when the power flow analysis run at minimum voltage levels of the distribution network when shunt admittances limits the operations of high voltage levels. The application of power analysis in a network grid is to post-process phase voltage angles through transformer phase shifting. Hence, the technique cannot be applied if the meshed grid network is weak. Therefore the transformer phase shifting model is now applied to control the operation of active power flow through power transformer in the loop. The phase shifting 
transformer model in analysis of power flow is very tedious problem which is difficult to represent by pi-equivalent symposium because of implicit asymmetric admittance matrix in the system [6].

There are various phase shifting transformer models with numerous application in many fields both transient and steady state operation $[2,15,19,21,24]$.

PSTs are constructional, a series connection of three phase transformers which generates a quadrature component voltage via a quadrature booster [14]. PST also enhances the power system stability by providing series compensation. The incorporation of the PST between buses is determined by the characteristics of the system. PSTs are quite effective on systems characterized by huge power transmission, a predominant direction of power flow and overloaded lines [9].

\subsection{Power System Analysis model}

In order to determine the state of the transmission system, it is crucial to ascertain the voltages and the entering power at difference buses. Thus, the voltage angle is always set to a reference bus number, which produce $4 \mathrm{~N}-1$ variable. When two voltages and injection power are unknown, there will be two balance equation of power i.e $2 \mathrm{~N}-1$ equations. Therefore, we must assign $2 \mathrm{~N}$ variables for the transmission system [7].

$$
\left[\operatorname{Vec}\left(R_{j}\right)\right]^{T}\left(y_{P F} \otimes y_{P F}\right)-\alpha=0 \quad \mathrm{~J}=1,2 \mathrm{~N}-1
$$

Where $\mathbf{R}$ and $\alpha$ are the matrix and the right-hand side, respectively regarding either the power injection or the

square of voltage magnitudes; and $y_{P F}=v$. From the equation (1) the system is overestimated, which means only $2 \mathrm{~N}-1$ which indicate one slack bus to analyze for the power system. Also v can be denoted as the optimization solution

$$
Y_{P F}^{S O L}=\arg _{\min }\left[\begin{array}{l}
\operatorname{Ve}\left(R_{1}\right)^{T} \\
\vdots \\
\operatorname{Vec}\left(R_{2 N-1}\right)^{T}
\end{array}\right]\left(y_{P F} \otimes y_{P F}\right)-\left(\begin{array}{l}
\alpha_{i} \\
\vdots \\
\vdots \\
\alpha_{2 N-1}
\end{array}\right)=\arg _{\min }\left(A_{P F}\left(y_{P F} \otimes y_{P F}\right)-b_{P F}\right)
$$

Hence, if solution in equation (1) and (2) are equal then

$$
A_{P F}\left(Y_{P F}^{S O L} \otimes Y_{P F}^{S O L}\right)-b_{P F}=0
$$

\subsection{Power Shifting Transformer}

The phase shifting transformers are usually used in power system for control of power flow in a transmission lines. The control of power flow is very important for determination of system market at different operators. Thus, phase shifting transformer (PST) gives total reliable and cheap solution for power flow control compared with FACTS devices [25]. Also, phase shifting transformers (PST) are uniquely designs and model with standard power transformers. These transformers are design for various applications but depend on the power system modes. They always design and model in single-core (direct) and two-core (indirect) designs. They also design based on the modes of operation either symmetrical or asymmetrical. In reference [23], the researcher design symmetrical system that changes the phase angle when the source magnitude and Load voltages are equal but asymmetrical changes the voltage magnitude and phase shift which also cause the tremendous changes in reactive power flow.

The active power flow in a transmission line is

$$
P=\frac{\left|V_{1}\right|\left|V_{2}\right|}{Z_{L}} \operatorname{Sin} \alpha
$$

Hence $V_{1}$ and $V_{2}$ are sending and receiving side voltages respectively, $\alpha$ is the phase shift between $V_{1}$ and $V_{2}$ and $\mathrm{Z}_{\mathrm{L}}$ is the reactance of the transmission line. The flow of the active power in the transmission line can be done by controlling the voltages [8][10]

\subsection{Materials and Method}

This paper present phase shifting transformer (PST) for controlling real power flow system in AC transmission lines using MATLAB 2015 software.

The paper analyze the effect of power system when PST is applied using IEEE 5 bus system. Also, NewtonRaphson load flow technique is employed in modeling the IEEE 5 bus system and the PST.

The PST shift the phase angle of the current and voltage and current which controls the operation of power 
flow in the transmission lines by varying the phase angle between the two buses. The changes in phase angle are connected to the component voltage source to line and to neural voltage [17]. The main purpose of PST is to change the current and phase angle of the voltage. Therefore the PST is connected in series with line between conjugate bus $(\mathrm{i}, \mathrm{j})$ which solves the load flow solutions[22].

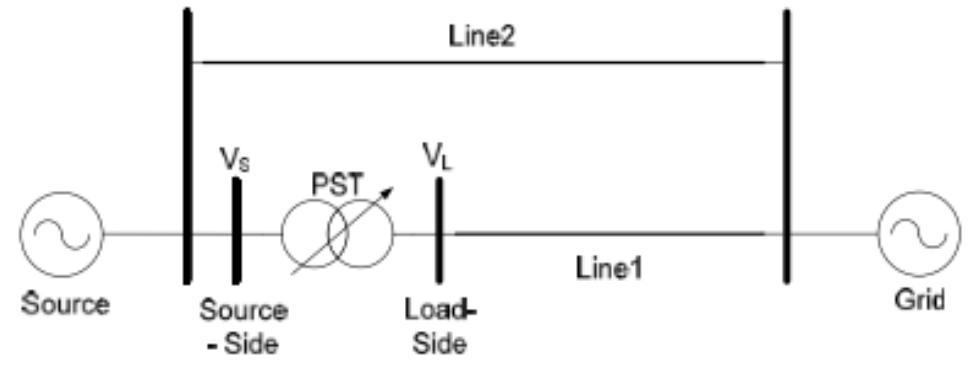

Figure 1a: Power flow control with Phase Shifting Transformer [18]

\subsection{Power Flow Control and Phase Shifter Adjustment}

The real power flow in a transmission line connected between any two buses in a power system is dependent on voltage phase angle difference between the two buses. To obtain a general equation, which relates the real power flow in a transmission line to a phase shift angle, assume a PST connected between bus $\mathrm{i}$ and $\mathrm{j}$ with an ideal turns ratio and shifting angle in series with the transformer admittance[18].

The procedures used in this paper are:

- Acquisition of line data and bus data of the IEEE 5 bus system

- Acquisition of PST data

- Modeling of the IEEE 5 bus system

- Identification of lines with low and high active power

- Incorporation of PST into the modeled system

\subsection{Power Flow Equation without PST}

The power injected, $\mathrm{S}_{\mathrm{GM}}$, into a bus $\mathrm{m}$, and the power supplied to a load by bus $\mathrm{m}$, are usually known. The difference between the injected power and supplied power at bus $\mathrm{m}$, is termed "Scheduled power". Thus, scheduled power at bus $m$ is given by:

$$
\begin{aligned}
& P_{m}^{s c h}=P_{G m}-P_{L m} \\
& Q_{m}^{s c h}=Q_{G m}-Q_{L m}
\end{aligned}
$$

Where $\mathrm{P}$ is the active power and $\mathrm{Q}$ the reactive power

The transmitted active and reactive power between the generating bus and load bus is denoted by $P_{m}^{\text {tran }}$ and $Q_{m}^{\text {tran }}$ respectively. Under a steady state operation, power mismatches $\Delta P_{m}$, and $\Delta Q_{m}$ is given by the equations (3) and (4):

$$
\begin{aligned}
\Delta P_{m} & =P_{m}^{s c h}-P_{m}^{\text {tran }} \\
\Delta Q_{m} & =Q_{m}^{s c h}-Q_{m}^{\text {tran }}
\end{aligned}
$$

This paper assumes that the bus voltages are not known, thus approximates values are proposed for the scheduled power. In this case, power mismatches are not taken to be zero. Hence, the improvement of bus voltages is carried out iteratively until convergence is obtained.

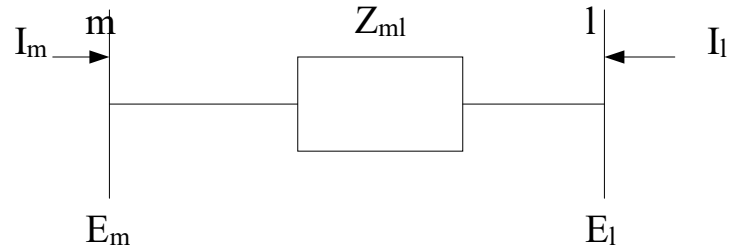

Figure 1b. Equivalent impedance circuit of a transmission line

The current flow between the bus $m$, and bus $l$ is given by equations (5) and (6)

$$
\begin{aligned}
& I_{m}=Y_{m l}\left(E_{m}-E_{1}\right) \\
& I_{m}=Y_{l m}\left(E_{1}-E_{m}\right)
\end{aligned}
$$

Where $Y_{\mathrm{ml}}$ and $Y_{1 \mathrm{~m}}$ are the bus admittance of line $\mathrm{m}$ an 1 .

The bus admittance matrix can be formulated as shown in equation (7) 


$$
\left[\begin{array}{c}
I_{m} \\
I_{l}
\end{array}\right]=\left[\begin{array}{cc}
Y_{m m} & -Y_{m l} \\
-Y_{l m} & Y_{l l}
\end{array}\right]\left[\begin{array}{c}
E_{m} \\
E_{l}
\end{array}\right]
$$

Voltages at bus $m$, and bus 1 are given by equation (8) and (9)

$$
\begin{aligned}
& \mathrm{E}_{\mathrm{m}}=\mathrm{V}_{\mathrm{m}}\left(\cos \delta_{\mathrm{m}}+\mathrm{j} \sin \delta_{\mathrm{m}}\right) \\
& \mathrm{E}_{\mathrm{m}}=\mathrm{V}_{\mathrm{l}}\left(\cos \delta_{1}+\mathrm{j} \sin \delta_{\mathrm{l}}\right)
\end{aligned}
$$

The complex power at bus $\mathrm{m}$ is given by equation (10)

$$
\mathrm{S}_{\mathrm{m}}=\mathrm{P}_{\mathrm{m}}+\mathrm{jQ} \mathrm{Q}_{\mathrm{m}}=\mathrm{E}_{\mathrm{m}} \mathrm{I}_{\mathrm{m}}{ }^{*}
$$

Where $\mathrm{I}_{\mathrm{m}}{ }^{*}$ is the complex conjugate of $\mathrm{I}_{\mathrm{m}}$

The power transmitted at bus $\mathrm{m}$ is given y equation (11):

$$
S_{m}^{\text {tran }}=\mathrm{V}_{\mathrm{m}}\left(\cos \delta_{\mathrm{m}}+\mathrm{j} \sin \delta_{\mathrm{m}}\right) \mathrm{I}_{\mathrm{m}}{ }^{*}
$$

The net transmitted active and reactive power at any bus $\mathrm{m}$ in the system is given by equations (12) and (13).

$$
\begin{aligned}
& P_{m}^{\text {tran }}=\sum_{i=1}^{m} P_{m}^{\text {itran }} \\
& Q_{m}^{\text {tran }}=\sum_{i=1}^{m} Q_{m}^{\text {itran }}
\end{aligned}
$$

The newton-Raphson solution to the power flow equation is represented by the Jacobian matrix below in equation (14).

$$
\left[\begin{array}{c}
\Delta P_{m} \\
\Delta P_{l} \\
\Delta Q_{m} \\
\Delta Q_{l}
\end{array}\right]=\left[\begin{array}{llll}
\frac{\partial P_{m}}{\partial \delta_{m}} & \frac{\partial P_{m}}{\partial \delta_{l}} & \frac{\partial P_{m}}{\partial V_{m}} V_{m} & \frac{\partial P_{m}}{\partial \delta_{l}} V_{l} \\
\frac{\partial P_{l}}{\partial \delta_{m}} & \frac{\partial P_{l}}{\partial \delta_{l}} & \frac{\partial P_{l}}{\partial V_{m}} V_{m} & \frac{\partial P_{l}}{\partial \delta_{l}} V_{l} \\
\frac{\partial Q_{m}}{\partial \delta_{m}} & \frac{\partial Q_{m}}{\partial \delta_{l}} & \frac{\partial Q_{m}}{\partial V_{m}} V_{m} & \frac{\partial Q_{m}}{\partial \delta_{l}} V_{l} \\
\frac{\partial Q_{l}}{\partial \delta_{m}} & \frac{\partial Q_{l}}{\partial \delta_{l}} & \frac{\partial Q_{l}}{\partial V_{m}} V_{m} & \frac{\partial Q_{l}}{\partial \delta_{l}} V_{l}
\end{array}\right]\left[\begin{array}{c}
\Delta \delta_{m} \\
\Delta \delta_{l} \\
\frac{\Delta V_{m}}{V_{m}} \\
\frac{\Delta V_{l}}{V_{l}}
\end{array}\right]
$$

The state variables are $\boldsymbol{\delta}_{\mathrm{m}}, \boldsymbol{\delta}_{1}, \mathrm{~V}_{\mathrm{m}}$ and $\mathrm{V}_{\mathrm{l}} \quad$ to which correction values $\Delta \boldsymbol{\delta}$ and $\Delta \mathrm{V}$ are added for each iteration. The derivatives of $\mathrm{P}$ and $\mathrm{Q}$ forms the Jacobian matrix's element. Iterations are started with initial estimates of state variables. The new voltage profile at bus $m$ is given by equations (15) and (16)

$$
\begin{aligned}
& V_{m}^{(i)}=V_{m}^{(i-1)}+\Delta V_{m}^{(i)} \\
& \delta_{m}^{(i)}=\delta_{m}^{(i-1)}+\Delta \delta_{m}^{(i)}
\end{aligned}
$$

Where ' $\mathrm{i}$ ' is the number of iteration

\subsection{Power Flow Equation with PST}

By incorporating PST in between buses $\mathrm{m}$ and 1 , the nodal current is given by equation (17)

$$
\left[\begin{array}{c}
I_{m} \\
I_{l}
\end{array}\right]=\left[\begin{array}{cc}
Y & -Y(\cos \alpha+j \sin \alpha) \\
-Y(\cos \alpha-j \sin \alpha) & Y
\end{array}\right]\left[\begin{array}{l}
V_{m} \\
V_{l}
\end{array}\right]
$$

Where $\alpha$ is the phase angle of the PST which varies within a range of ( $\alpha \min <\alpha<\alpha$ max $)$. The power mismatch equation is represented by the Jacobian matrix in equation (18)

$$
\left[\begin{array}{c}
\Delta P_{m} \\
\Delta P_{l} \\
\Delta Q_{m} \\
\Delta Q_{l} \\
\Delta P_{m l}^{(\alpha P S)}
\end{array}\right] \quad=\left[\begin{array}{ccccc}
\frac{\partial P_{m}}{\partial \delta_{m}} & \frac{\partial P_{m}}{\partial \delta_{l}} & \frac{\partial P_{m}}{\partial V_{m}} V_{m} & \frac{\partial P_{m}}{\partial V_{l}} V_{l} & \frac{\partial P_{m}}{\partial \alpha P S} \\
\frac{\partial P_{l}}{\partial \delta_{m}} & \frac{\partial P_{l}}{\partial \delta_{l}} & \frac{\partial P_{l}}{\partial V_{m}} V_{m} & \frac{\partial P_{l}}{\partial V_{l}} V_{l} & \frac{\partial P_{l}}{\partial \alpha P S} \\
\frac{\partial Q_{m}}{\partial \delta_{m}} & \frac{\partial Q_{m}}{\partial \delta_{l}} & \frac{\partial Q_{m}}{\partial V_{m}} V_{m} & \frac{\partial Q_{m}}{\partial V_{l}} V_{l} & \frac{\partial Q_{m}}{\partial \alpha P S} \\
\frac{\partial Q_{l}}{\partial \delta_{m}} & \frac{\partial Q_{l}}{\partial \delta_{l}} & \frac{\partial Q_{l}}{\partial V_{m}} V_{m} & \frac{\partial Q_{l}}{\partial V_{l}} V_{l} & \frac{\partial Q_{l}}{\partial Q_{l}} \\
\frac{\partial P_{m l}^{(\alpha P S)}}{\partial \delta_{m}} & \frac{\partial P_{m l}^{(\alpha P S)}}{\partial \delta_{l}} & \frac{\partial P_{m l}^{(\alpha P S)}}{\partial V_{m}} V_{m} & \frac{\partial P_{m l}^{(\alpha P S)}}{\partial V_{m}} V_{l} & \frac{\partial P_{m l}^{(\alpha P S)}}{\partial \alpha P S}
\end{array}\right]\left[\begin{array}{c}
\Delta \delta_{m} \\
\Delta \delta_{l} \\
\frac{\Delta V_{m}}{V_{m}} \\
\frac{\Delta V_{l}}{V_{l}} \\
\Delta \alpha P S
\end{array}\right]
$$

Where $\Delta P_{m l}^{(\alpha P S)}$ is the active power mismatch for the phase shifting transformer which is expressed in equation (19)

$$
\Delta P_{m l}^{(\alpha P S)}=P_{m l}^{(\alpha r e g)}-P_{m l}^{(\alpha P S)}
$$

The updated angle of the phase shifter is expressed in equation (20)

$$
\alpha_{m}^{(i)}=\alpha_{m}^{(i-1)}+\Delta \alpha_{m}^{(i)}
$$

For each iteration, the phase shifter is checked to ascertain that it has not exceeded the specified limit beyond which it will be incapable of controlling active power.

This paper uses a phase angle range of $-20^{0}$ to $20^{\circ}$ in order to keep the difference in input and output voltage at considerable value.

\subsection{RESULTS AND DISCUSSION}

Simulation was carried out using the MATLAB Toolbox. The bus data available from the IEEE 5 Bus system are the generator buses and load buses. There are two generator buses in the system which is bus 1 and bus 2 . Bus 1 , 
having the highest generated power was taken as the slack bus with its voltage at 1.06 p.u. and a phase angle of zero degree. The three other buses namely bus 3, 4, and 5 forms the load buses.

\section{(a) Power Flow Solution without PST}

Steady state simulation of the IEEE 5 bus system without PST was carried out. A tolerance of $1.000 \mathrm{e}^{-12}$ was used and convergence was reached after 6 iterations. Tables 1 presents the power flow solution and table 2 present the line flows and losses.

\begin{tabular}{|l|l|l|l|l|l|l|}
\hline Bus no & Voltage magnitude (p.u) & Phase & \multicolumn{2}{l|}{ PQ GENERATOR } & \multicolumn{2}{l|}{ PQ LOAD } \\
\cline { 4 - 7 } & & Angle (degree) & MW & Mvar & MW & Mvar \\
\hline 1 & 1.06 & 0 & 131.12 & 90.82 & 0 & 0 \\
\hline 2 & 1 & -2.0612 & 40 & 61.5 & 20 & 10 \\
\hline 3 & $\mathbf{0 . 9 8 7 2}$ & -4.6367 & 0 & 0 & 45 & 15 \\
\hline 4 & 0.9841 & -4.957 & 0 & 0 & 40 & 5 \\
\hline 5 & 0.9717 & -5.7649 & 0 & 0 & 60 & 10 \\
\hline
\end{tabular}

Table 1. Power flow solution without PST

\begin{tabular}{|r|r|r|r|r|r|r|r|r|}
\hline \multicolumn{1}{|l|}{ s/n } & fb & tb & \multicolumn{2}{|c|}{ PQ SEND } & \multicolumn{2}{|c|}{ PQ REC } & \multicolumn{2}{c|}{ LINE LOSSES } \\
\hline & & & MW & \multicolumn{1}{l|}{ Mvar } & MW & Mvar & MW & Mvar \\
\hline 1 & 1 & 2 & 89.33 & 74 & 86.85 & 72.87 & 2.48 & 1.13 \\
\hline 2 & 1 & 3 & 41.79 & 16.82 & 40.32 & 17.2 & 1.47 & -0.38 \\
\hline 3 & 2 & 3 & 24.74 & -2.52 & 24.06 & 0.45 & 0.68 & -2.97 \\
\hline 4 & 2 & 4 & 28.1 & 1.12 & 27.41 & 0.95 & 0.69 & 0.17 \\
\hline 5 & 2 & 5 & 54.73 & 5.53 & 53.01 & 4.65 & 1.72 & 0.88 \\
\hline 6 & 3 & 4 & 19.45 & 2.63 & 19.24 & 4.7 & 0.21 & -2.07 \\
\hline 7 & 4 & 5 & 6.89 & 0.45 & 6.34 & 4.89 & 0.55 & -4.44 \\
\hline
\end{tabular}

Table 2. Line flows and losses without PST

Table 2 shows that line 1-2 has the largest active power transfer with $89.33 \mathrm{MW}$ at the end and $86.85 \mathrm{MW}$ at the receiving end. Line 4-5 transfers the lowest active power in the system with $6.89 \mathrm{MW}$ at the sending end and $6.34 \mathrm{MW}$ at the receiving end. The total active power loss in the system is $7.8 \mathrm{MW}$ and the total reactive power loss is -7.68 MVar.

\section{(b) Power Flow Solution with PST}

The PST was placed between bus 3 and bus 4 .. The phase angle of the PST was set at $-6.1^{0}$ and bus 3 is the controlled bus. Simulation was carried out using the MATLAB software. A tolerance of $1.000 \mathrm{e}^{-12}$ was used and convergence was reached after 5 iterations. The results are shown in tables 3 and 4 .

\begin{tabular}{|l|l|l|l|l|l|l|}
\hline \multirow{2}{*}{ Bus no } & Voltage magnitude (p.u) & Phase & PQ GENERATOR & \multicolumn{2}{l|}{ PQ LOAD } \\
\cline { 4 - 7 } & & Angle (degree) & MW & Mvar & MW & Mvar \\
\hline 1 & 1.06 & 0 & 132.5 & 90.72 & 0 & 0 \\
\hline 2 & 1 & -2.0612 & 42 & 62.05 & 20 & 10 \\
\hline 3 & 0.9874 & -4.6367 & 0 & 0 & 45 & 15 \\
\hline 4 & 0.9844 & -4.957 & 0 & 0 & 40 & 5 \\
\hline 5 & 0.9714 & -5.7649 & 0 & 0 & 60 & 10 \\
\hline
\end{tabular}

Table 3. Power flow result with PST

\begin{tabular}{|r|r|r|r|r|r|r|r|r|}
\hline $\mathrm{s} / \mathrm{n}$ & $\mathrm{fb}$ & \multicolumn{1}{|c|}{ tb } & \multicolumn{2}{|c|}{ PQ SEND } & \multicolumn{2}{c|}{ PQ REC } & \multicolumn{2}{c|}{ LINE LOSSES } \\
\hline & & & \multicolumn{1}{c}{ MW } & Mvar & MW & Mvar & MW & \multicolumn{1}{l|}{ Mvar } \\
\hline 1 & 1 & 2 & 82.41 & 77.4 & 81.3 & 76.2 & 1.11 & 1.2 \\
\hline 2 & 1 & 3 & 52.72 & 17.45 & 49.98 & 16.3 & 2.74 & 1.15 \\
\hline 3 & 2 & 3 & 38.1 & -3.95 & 37.7 & 4.3 & 0.4 & -8.25 \\
\hline 4 & 2 & 4 & 14 & 3.1 & 13.8 & 5.8 & 0.2 & -2.7 \\
\hline 5 & 2 & 5 & 47.7 & 7.3 & 46.95 & 5.3 & 0.75 & 2 \\
\hline 6 & 3 & 4 & 40 & 3.92 & 39.6 & 2.6 & 0.4 & 1.32 \\
\hline 7 & 4 & 5 & 14.6 & -1.5 & 13.9 & 2.34 & 0.7 & -3.84 \\
\hline
\end{tabular}

Table 4. Line flows and losses with PST

It is evident from table 4 that there is a redistribution of power on lines across the entire power system. There is a decrease in active power flow on line 1-2 from 89.33MW without PST to 82.41MW with PST. Other lines 
with decreased active power flow are line $2-5$ from $54.73 \mathrm{MW}$ to $47.7 \mathrm{MW}$ and line $2-4$ from $27.71 \mathrm{MW}$ to $14.0 \mathrm{MW}$. On contrary, lines with low active power flow are seen to increase in load ability with line 1-3 increasing from 41.79MW to $52.72 \mathrm{MW}$, line $2-3$ from $24.74 \mathrm{MW}$ to $38.1 \mathrm{MW}$, line $3-4$ from 19.45 to $40.0 \mathrm{MW}$ and line $4-5$ from 6.89MW to $14.60 \mathrm{MW}$. Figure 1 compares the active power at the sending end of the lines without PST and with PST.

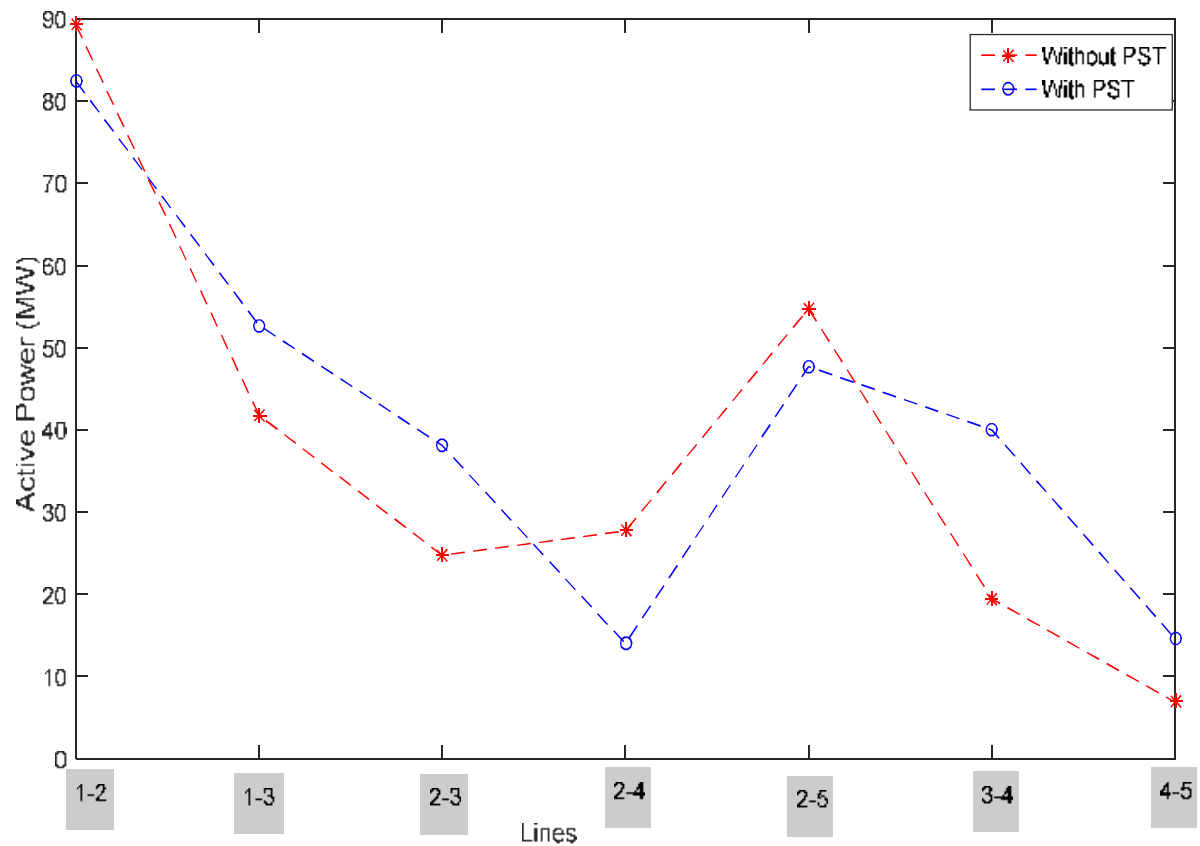

Figure 1. Power flow on lines without and with PST

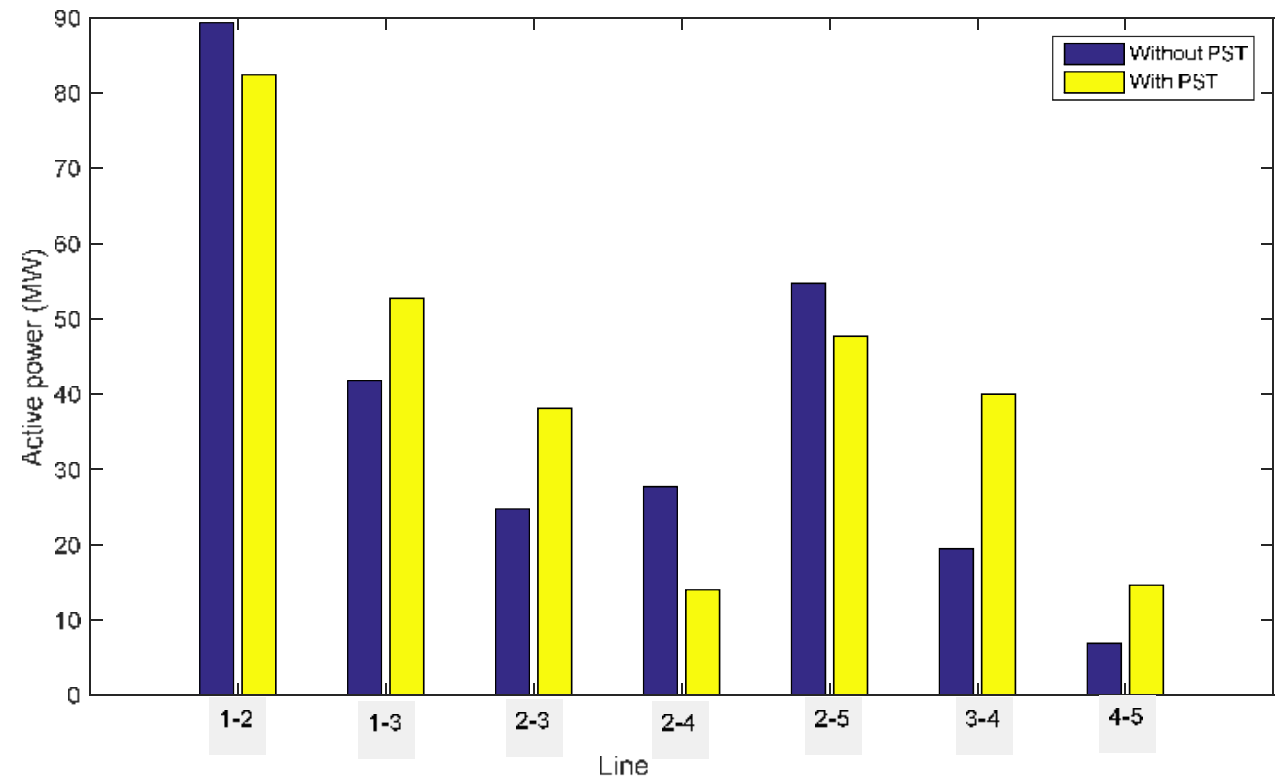

Figure 2. Bar graph Power flow on lines without and with PST

Figures 3 and 4, compares the line losses of active power without PST and with PST. 


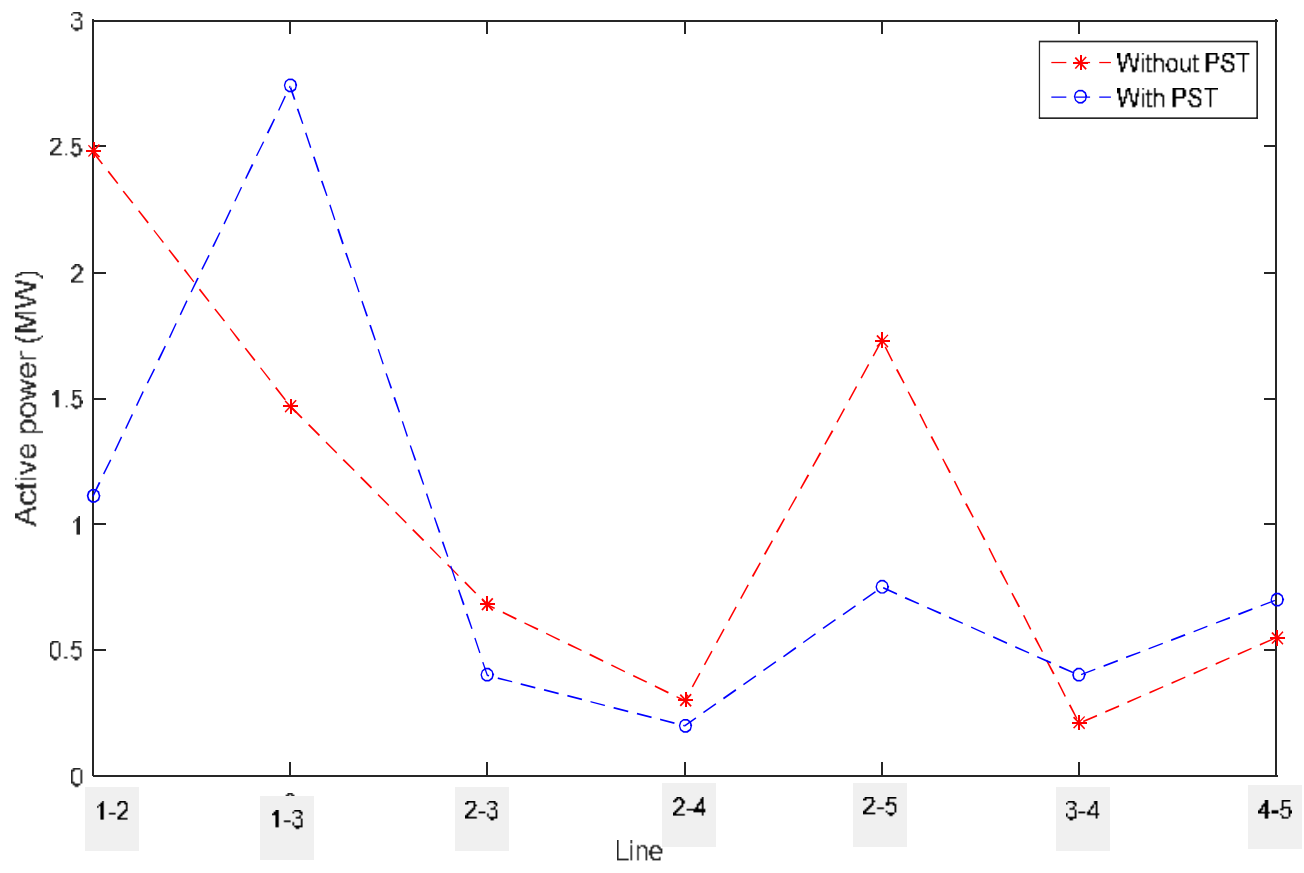

Figure 3. Graph showing power flow line losses without and with PST

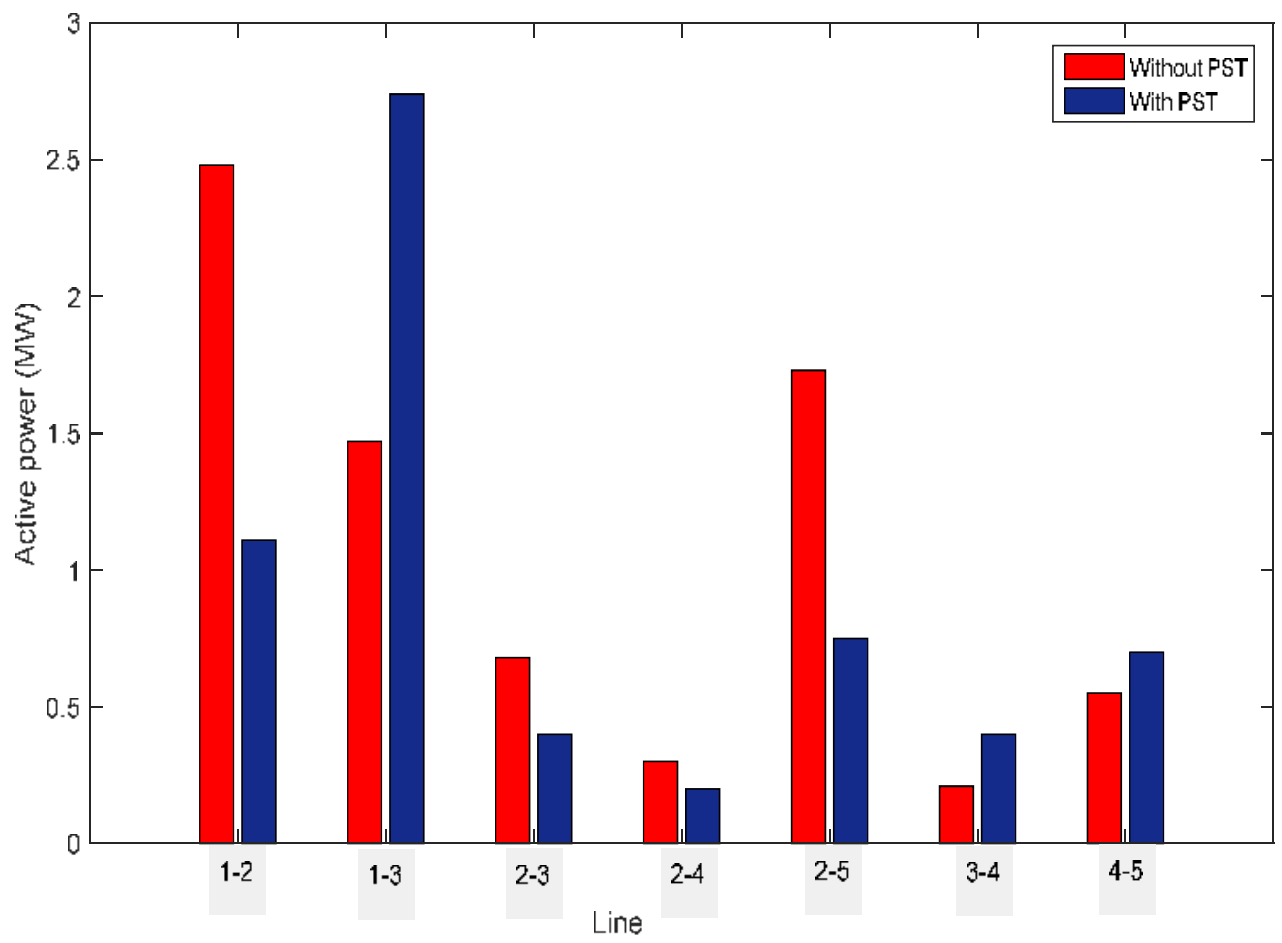

Figure 4. Bar graph showing power flow line losses without and with PST

It is shown from table 4 and figures 3 and 4 that with PST, lines 1-2, 2-3, 2-4, and 2-5 have reduced line losses from $2.48 \mathrm{MW}$ to $1.11 \mathrm{MW}, 0.68 \mathrm{MW}$ to $0.4 \mathrm{MW}, 0.3 \mathrm{MW}$ to $0.2 \mathrm{MW}$ and $1.73 \mathrm{MW}$ to $0.75 \mathrm{MW}$ respectively. Lines 1-3,3-4 and 4-5, experienced increase in line losses from $1.47 \mathrm{MW}$ to $2.74 \mathrm{MW}, 0.21 \mathrm{MW}$ to $0.4 \mathrm{MW}$ and $0.55 \mathrm{MW}$ to $0.7 \mathrm{MW}$ respectively. The overall active power loss with PST is $6.3 \mathrm{MW}$ compared with $7.8 \mathrm{MW}$ without PST. It is shown that there is a reduction of active power loss in the system with the incorporation of PST between bus 3 
and 4.

\section{CONCLUSION}

In this paper, power flow analysis was carried out using Newton-Raphson method with MATLAB simulation. Power flow and line losses were obtained under the steady state condition. Some lines were seen to be overloaded while others were less loaded. PST was inserted on a suitable line with its phase angle varied. This resulted in redistribution of active power on the lines, thus, controlling overload and reduced the system' total power loss. Hence, it is affirmed that phase shifting transformers, when placed between appropriate buses are capable of controlling power flow, reducing congestion, and improve the overall efficiency of the power system.

\section{REFERENCES}

[1]ABB Review: FACTS - Solutions to power flow control \& stability problems. Sweden: ABB Power Systems AB, 1999, 16p.

[2]Acha E, Ambriz-Perez H, Fuerte-Esquivel CR. (2000) Advanced transformer control modeling in an optimal power flow using Newton's method. IEEE Trans Power Syst ;15(1):290-8.

[3] Bandler J, El-Kady M, Grewal H. (1999) An application of complex branch modeling to nonreciprocal power transmission elements. IEEE Trans Circ Syst 1985;32 (12):1292-5.

[4] Cano JM, Norniella JG, Rojas CH, Orcajo GA, Jatskevich J. (2015). Application of loop power flow controllers for power demand optimization at industrial customer sites. In: IEEE power energy society general meeting. p. $1-5$.

[5] Das D, Kothari DP, Kalam A. (1995). Simple and efficient method for load flow solution of radial distribution networks. Int J Electr Power Energy Syst.17 (5):335-46.

[6] Gómez-Expósito A, Conejo AJ, Cañizares C (2009). Electric energy systems: analysis and operation. 1st ed. Boca Raton, FL: CRC Press LLC.

[7] Hyungseon, (2019)“A unified and Efficient Approarch to power flow Analysis"department of electrical and computer engineering NAVAL Academy USA.

[8] IEEE Std C57.135 (2001) "IEEE Guide for the Application, Specification and Testing of Phase Shifting Transformers".

[9] J. Ptacek, P. Modlitba, S. Vnoucek and J. Cermak (2006), "Possibilities of applying Phase shifting Transformers in the electric power system of the Czech Republic", cigre.

[10] James. H. H, (2004) :Electric Power Transformer Engineering”, CRC Press LLC.

[11] Jimenez JC, Nwankpa CO. (2011). Circuit model of a phase-shifting transformer for analog power flow emulation. In: IEEE international symposium of circuits and systems (ISCAS). p. 1864-7.

[12] Kersting WH. (2002) Distribution system modeling and analysis. 1st ed. Boca Raton, FL: CRC Press LLC.

[13] Martinez JA, Mahseredjian J. (2011). Load flow calculations in distribution systems with distributed resources: a review. In: 2011 IEEE power and energy society general meeting. p. 1-8.

[14] Mohammed Ali AL-Rawi and Mohammed Obiad Mostafa (2010), "Power Flow Control Using Phase Shifting Transformer", Tikrit Journal of Eng. Sciences/Vol.17/No.1/March 2010, (10-15)

[15] Nabavi Niaki SA. A novel steady-state model and principles of operation of phase-shifting transformer comparable with FACTS new devices. Proceedings of international conference on power system technology, vol. 3. p. 1450-7.

[16] Paul E. Rylatt, George J. Cokkin-ides, (1989)“A method for planning Phase Shif ting Transformers Using Linearized Network Sensitivities”, IEEE proceding-1989 southeaston, pp. 832-835.

[17] R.D. Youssef, (1993) "Phase-Shifting Trans- formers in Load Flow and Short Ciruit Analysis: Modelling and Control", IEEE Proceedings - C, Vol. 140, No. 4, July 1993.

[18] R. Grunbaum, R. Sharma, and J. P. charpentier, (2000) "Improving the efficiency and quality of A.C transmission system", ABB power system- Joint world Bank, Tech. Rep.

[19]Shirmohammadi D, Hong HW, Semlyen A, Luo GX. (1988). A compensation-based power flow method for weakly meshed distribution and transmission networks. IEEE Trans Power System 3(2):7 53-62.

[20]Teng J-H. A direct approach for distribution system load flow solutions. IEEE Trans Power Deliv 2003;18(3):882-7.

[21]Tziouvaras DA, Jimenez R. (2004). $138 \mathrm{kV}$ phase shifting transformer protection: EMTP modeling and model power system testing. 2004 Eighth IEE international conference on developments in power system protection, vol. 1. p. 343-7.

[22]UCTE, "Union for the coordination ordination of Transmission of Electricity, "UCTE system Adequacy Retrospect 2002", Available :http//www.ucte.org/publicationslibrary/e_de fault_2003.asp

[23] Verboomen J., Hertem D. V., Schavemaker P. H., Kling W. L., Belmans R., (2005) "Phase Shifting Transformer: Methods and Applications", in Proc. Intl. IEEE Conf. on Future Power Systems., Amsterdam, Netherland, Nov 2005, p. 6. 
[24] Youssef RD. (1993). Phase-shifting transformers in load flow and short-circuit analysis: modelling and control. IEE Proc C - Gener Transm Distrib 1993;140 (4):331-6.

[25] Zhang X. P., Rehtanz C., Pal B., "Flexible AC Transmission System: Modeling and Control”, Germany: Springer; 2006 